\title{
FOOD REGION DETECTION USING BAG-OF-FEATURES REPRESENTATION AND COLOR FEATURE
}

\author{
Ruiko Miyano, Yuko Uematsu and Hideo Saito \\ Keio University, 3-14-1 Hiyoshi, 223-8522 Kohoku-ku, Japan \\ \{rui, yu-ko, saito\}@hvrl.ics.keio.ac.jp
}

Keywords: $\quad$ Food Region Detection, Bag-of-Features, Visual Words, Color Feature, SURF, Support Vector Machine.

\begin{abstract}
Food image processing has recently attracted attention, because many people take photos of food. For food image processing, recognition of captured food is an essential technology, but region detection of the food area from captured photos is also very important procedure for food recognition. In this paper, we propose a novel method for automatic region detection of food from photos using two kinds of features in input image. To detect food regions, we use a method which is widely used in generic object recognition. We divide an image into small subregions and represent each subregion as Bag-of-Features representation using local feature descriptors and color feature. Using two features, we recognize food subregions and finally connect them as food regions. Our experiments show that the proposed method can detect food region in high accuracy.
\end{abstract}

\section{INTRODUCTION}

In recent years, the number of camera users has increased because digital cameras are very popular consumer products. Since a human face is the most popular subject captured with the consumer digital cameras, techniques for face detection have been extensively developed. As well as human faces, food is also targeted when recording food logs or memorizing special moments of travel. Additionally, there are some applications which make food appearance more delicious in a photo. For these reasons, food is the subject frequently taken in everyday life.

Some researches dealing with food images have attracted attention in recent years. Especially, automatic food image recognition is important as the generic object recognition. Such systems aim to classify unknown food images and estimate volume, nutrition and so on. Using these systems, people who often take a photo of food can easily analyze their photos, and people can easily record everyday food from photos.

However, these researches have some problems. In these researches, food is located in the entire screen or food region is estimated based on circle detection. When there are several plates in the captured image, therefore, they have to cut out the regions of food in advance. Moreover, if the plate does not have circle shape, plate detection based on circle detection will fail. Therefore, we aim to automatically and precisely detect food regions from single image.

\section{RELATED RESEARCH}

Joutou et al. proposed a food image recognition system for 50 kinds of food images (Joutou and Yanai, 2009). They extract Bag-of-Features (BOF), color histogram and Gabor texture feature, and then apply Multiple Kernel Learning (MKL) method to those features in order to classify query images into 50 categories. Yang et al. proposed a fast food image recognition system (Yang et al., 2010). They use pairwise statistics which represents geometric relationship such as distance and orientation between many pairs of local features. Puri et al. proposed a food intake assessment system which recognizes food types and estimates volumes and nutrition information (Puri et al., 2009). They recognize food types by using color feature and texture feature, and estimate volumes by $3 \mathrm{D}$ reconstruction.

There are many researches about object detection. Most popular research is face detection ( $\mathrm{Vi-}$ ola and Jones, 2004). Face detection is implemented in most digital cameras, which is used to lock focus and adjust flash. To detect face region, they use Haar-like feature which considers characteristic patterns of human faces. On the other hand, food images do not have such particular characteristic patterns because foods have several colors, shapes and ingredients. Therefore, we focus on a method used in generic object recognition. To detect object region based on generic object recognition, it is useful to use subwindow (Lampert et al., 2009; Wei and Tao, 2010). 
In generic object recognition, we need to compare a query image to pre-trained images. To effectively compare them, the images have to be represented as low-dimensional data. The BOF representation (Csurka et al., 2004) using local feature descriptors and color information (Swain and Ballard, 1991) are most general methods.

In our proposed method, we do not recognize types of foods but detect regions where the food is located in a single photo. We employ sliding window approach that slides target subregion to detect food regions. For recognizing a subregion as food or not, BOF representation and color feature are applied.

\section{PROPOSED METHOD}

In this paper, we propose a method to automatically detect food regions from a single image. Fig. 1 shows the overview of our proposed method. Our method consists of two phases: training and detection.

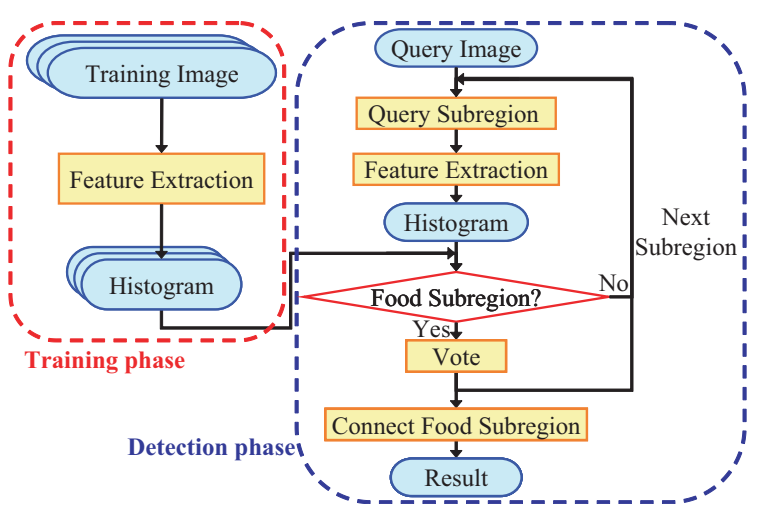

Figure 1: Overview.

In training phase, we extract two kinds of image features, which are BOF representation and color feature, from training images (730 food images). The food categories which can be detected depend on training images. We collected eight kinds of food, which are pasta, salad, curry, hamburger, ramen, pizza, rice omelet and okonomiyaki.

In detection phase, we cut out a subregion from a query image as a query subregion, and extract two image features from the query subregion in the same way as training phase. The size of a subregion is $200 \times 200$ pixels and we slide a subregion with 20 pixels. The subregion recognized as food using the image features is called a food subregion. We slide a query subregion around the query image and connect only food subregions as food region in the query image.

\subsection{Features}

In this section, we explain two kinds of image features. In contrast with human face images, food images do not have characteristic patterns. Therefore we combine BOF representation using local feature descriptors, and color feature in the image.

\subsubsection{Bag-of-Features Representation}

Local characteristic patterns in images are important in generic object recognition. To utilize local characteristic patterns, the BOF representation is widely used. In this representation, an image is represented as the distribution of feature descriptor vectors.

In training phase, we extract SURF from training images, which are 100 food images and 100 non food images, and cluster all feature points into 500 clusters using K-Means++ method. Then we define the centroids of 500 clusters as Visual Words.

We compute histogram of Visual Words in both training and detection phase. We extract SURF from training image or query subregion. Then we quantize each feature point into 500 Visual Words (Fig. 2(a)) and count the number of occurrences (Fig. 2(b)). In Fig. 2, the different visual words are represented by different colors for the detected feature points. Finally, we normalize histogram using $t f$-idf weighting. In this way, we compute Visual Word histogram, which is so-called BOF representation.

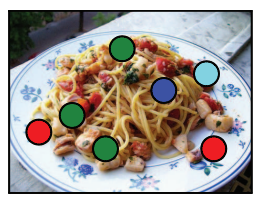

(a) Visual Words

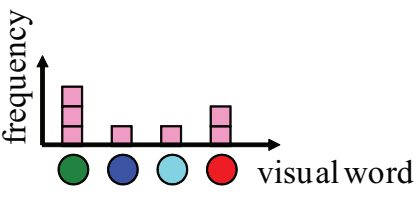

(b) Histogram of visual words
Figure 2: BOF representation.

\subsubsection{Color Feature}

Not only local feature points but also color information is important in food images, because most of foods consists of ingredients that have specific colors.
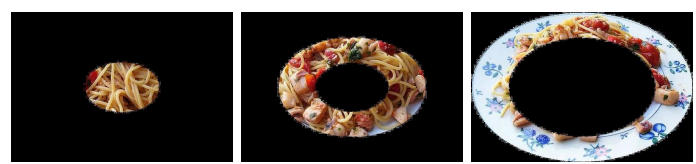

Figure 3: Three parts for color feature.

To extract color feature, we divide an image into 3 parts concentrically as shown in Fig. 3. This is because most foods are placed in a circle. We also divide 
RGB brightness into 4 parts. Then we create 64-bin color histogram from each parts. Totally, we extract a 192 dimensional color feature from each image.

\subsection{Recognition}

In this section, we explain the method to recognize subregions as food in a query image. For the recognition, our method compares two image features between each query subregion and training images. We propose two methods to compare these image features. One is histogram intersection and the other is Support Vector Machine (SVM).

\subsubsection{Histogram Intersection}

First, we explain the method to compute similarity of image features using histogram intersection (Swain and Ballard, 1991). We compute the histogram intersection for each histogram between the query subregion and the training images, and compute the average of two histogram intersections. If the average is larger than a pre-defined threshold, these two images are regarded as similar images.

We calculate histogram intersection between one query subregion and all training images, and count the number of similar images. If the number of similar images is larger than a pre-defined threshold, the query subregion is recognized as food subregion.

\subsubsection{Support Vector Machine}

Second, we explain the method to classify query subregions into categories of food using SVM. SVM is one of the common classification methods that has high generalization performance, even the dimension of data is very high. The simplest SVM can classify data into two classes. It needs known positive data and negative data, and estimates hyperplane which correctly divides these data into two classes.

In training phase, we create 2-class SVM classifier for each food category and each feature using training images, which are 730 food images and 100 non food images. In detection phase, we predict class of query subregion using SVM classifiers created in training phase.

\subsection{Connection of the Food Subregions}

In this section, we explain the method to connect the food subregions.

In section 3.2, we recognize several food subregions by the sliding window recognition. We vote for all pixels included in the food subregions as show in Fig. 4(a). If the voted count of each pixel is larger

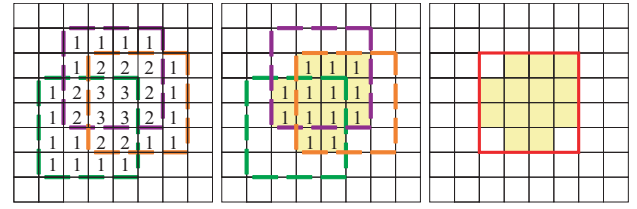

(a)

(b)

(c)

Figure 4: Connection of the food subregions.

than a pre-defined threshold, that pixel is regarded as inside the food region, which is labeled as shown in Fig. 4(b). Finally, as shown in Fig. 4(c), we draw the bounding box of those pixels.

\section{EXPERIMENTS}

We carried out 2 experiments, which are evaluation of two features and detection of food regions, to evaluate our proposed method.

\subsection{Evaluation of Two Features}

In this experiment, we use 100 food images and 50 non food images as query images in order to evaluate the effectiveness of two features; BOF representation and color feature. Here, we do not cut out query subregions via sliding window but create histograms of a whole image and recognize a query image as food or non food.

Table 1: The recognition results.

\begin{tabular}{|c|c|c|c|}
\hline & \multicolumn{2}{|c|}{ Correct answers } & \multirow{2}{*}{$\begin{array}{c}\text { Accuracy } \\
\text { rate }\end{array}$} \\
\cline { 2 - 3 } & Food & Non food & \\
\hline \hline BOF (HI) & $91 / 100$ & $40 / 50$ & $87.3 \%$ \\
\hline Color (HI) & $90 / 100$ & $34 / 50$ & $82.7 \%$ \\
\hline Both (HI) & $96 / 100$ & $38 / 50$ & $89.3 \%$ \\
\hline \hline BOF (SVM) & $98 / 100$ & $30 / 50$ & $85.3 \%$ \\
\hline Color (SVM) & $100 / 100$ & $33 / 50$ & $88.7 \%$ \\
\hline Both (SVM) & $98 / 100$ & $50 / 50$ & $98.7 \%$ \\
\hline
\end{tabular}

Table 1 shows the recognition results using both approaches. It seems the combination of BOF representation and color feature is effective because the accuracy rates using both two features is $89.3 \%$ and $98.7 \%$ which is larger than the results with a single feature. We can also find that SVM approach is better than histogram intersection approach because it has general versatility. Histogram intersection approach depends on training images because we compare a query subregion and all training images. Therefore it cannot be applied to data which is not included in training images. 


\subsection{Detection of Food Regions}

In this section, we show the result of food region detection. We compare three methods, which are circle detection, histogram intersection and SVM. Histogram intersection and SVM are based on the method explained in section 3.2. Circle detection is used in Puri's research (Puri et al., 2009). We recognize detected circular regions as food after detecting circle by Hough transform.

To compare them, we compute difference between ground truth images and each detected image. Fig. 5(b) shows manually created ground truth image of Fig. 5(a). Fig. 5(c) shows a mask of detection result. And Fig. 5(d) shows the difference between Fig. 5(b) and Fig. 5(c). Red region represents false negative, and green region represents false positive.

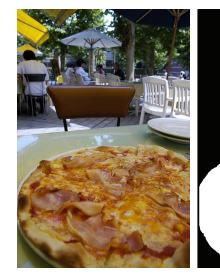

(a)

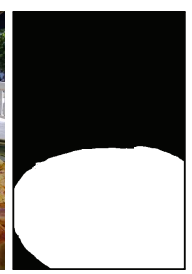

(b)

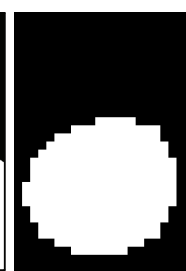

(c)

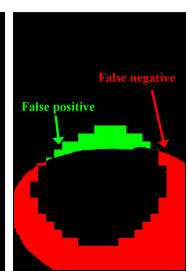

(d)
Figure 5: Computation of difference.

We computed false detection rate $R$ according to following equation

$$
R=\frac{\text { false negative }+ \text { false positive }(\text { pixel })}{\text { width } \times \text { height }(\text { pixel })} .
$$

If ground truth image is same as detection result, difference is $0 \%$.

Table 2 shows the average of false detection rates between each ground truth image and each detection result (389 food images and 15 non food images).

Table 2: False detection rate.

\begin{tabular}{|c|c|c|c|}
\hline & $\begin{array}{c}\text { Circle } \\
\text { detection }\end{array}$ & $\begin{array}{c}\text { Histogram } \\
\text { intersection }\end{array}$ & SVM \\
\hline \hline$R$ & $35.5 \%$ & $29.2 \%$ & $25.4 \%$ \\
\hline
\end{tabular}

Fig. 6 shows the examples of detection result. Fig. 6(a) are based on histogram intersection approach. Fig. 6(b), 6(c) are based on SVM approach. We can see that food regions of our eight categories are almost detected from these images. We compare three methods in Fig. 7. The food region can be detected using histogram intersection and SVM, but it cannot be detected using circle detection. In circle detection, it depends on a shape of plate. For example, if the plate is square, or whole plate is not in query image

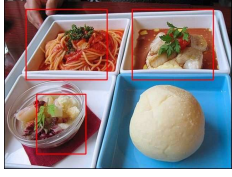

(a)

Figure 6: Food region detection.

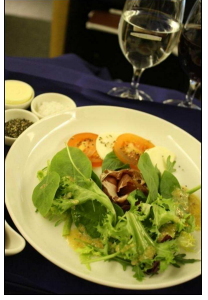

(a) Circle detection

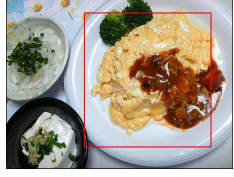

(b)

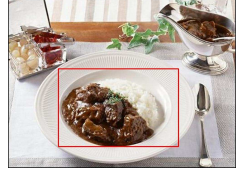

(c)

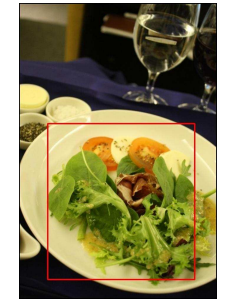

(b) Histogram intersection

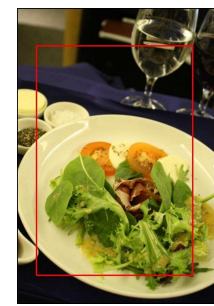

(c) SVM
Figure 7: Comparison of three methods.

like Fig. 7(a), detection will be fail. Therefore, our sliding window approach is efficient, even the plate has non circular shape.

\section{CONCLUSIONS}

We proposed an automatic detection method of food regions from a single image. By using BOF representation and color feature, we recognized subregions as food or non food, and then all food subregions are connected to create food regions.

We carried out two experiments to evaluate our proposed method. First, we recognized whole query image to evaluate the effectiveness of two features. Because of combining two features, we achieved 98.7 $\%$ accuracy rate based on SVM approach. Second, we applied our method to query images. We can automatically detect food regions from query images due to sliding window approach, and false detection rate is $25.4 \%$ based on SVM approach.

As future works, we plan to add more features to increase recognition accuracy, improve the method for connecting food subregions and extend training images to increase categories of food.

\section{ACKNOWLEDGEMENTS}

A part of this research was supported by the Research Grant of Keio Leading-edge Laboratory of Science \& Technology. 


\section{REFERENCES}

Csurka, G., Dance, C. R., Fan, L., Willamowski, J., and Bray, C. (2004). Visual categorization with bags of keypoints. In Workshop on statistical learning in computer vision, ECCV, volume 1, pages 1-22.

Joutou, T. and Yanai, K. (2009). A food image recognition system with multiple kernel learning. In Proc. of ICIP.

Lampert, C. H., Blaschko, M. B., and Hofmann, T. (2009). Efficient subwindow search: A branch and bound framework for object localization. IEEE Trans. on PAMI, 31(12):2129-2142.

Puri, M., Zhu, Z., Yu, Q., Divakaran, A., and Sawhney, H. S. (2009). Recognition and volume estimation of food intake using a mobile device. In Proc. of WACV, pages $1-8$.

Swain, M. J. and Ballard, D. H. (1991). Color indexing. International Journal of Computer Vision, 7(1):11-32.

Viola, P. A. and Jones, M. J. (2004). Robust real-time face detection. International Journal of Computer Vision, 57(2):137-154.

Wei, Y. and Tao, L. (2010). Efficient histogram-based sliding window. In Proc. of CVPR, pages 3003-3010.

Yang, S., Chen, M., Pomerleau, D., and Sukthankar, R. (2010). Food recognition using statistics of pairwise local features. In Proc. of CVPR, pages 2249-2256. 